\title{
Risk Factors for Plantar Fasciitis Among Assembly Plant Workers
}

\author{
Robert A. Werner, MD MS, Nancy Gell, MPH PT, Anne Hartigan, MD, \\ Neal Wiggerman, MS, William M. Keyserling, PhD
}

Objective: The objective of this study was to determine the relative contributions of work activity (time spent standing, walking, or sitting), floor surface characteristics, weight, body mass index, age, foot biomechanics, and other demographic and medical history factors to the prevalence of plantar fasciitis.

Design: A cross-sectional observational study design was used.

Setting: The study site was an automobile engine assembly plant.

Participants: Full-time employees of the assembly plant who had been working at least 6 months.

Assessment of Risk Factors: The independent variables included baseline demographics, medical history, ergonomic exposures, psychosocial factors, discomfort ratings, shoe characteristics, and foot biomechanics.

Main Outcome Measurements: The dependent variable was the finding of plantar fasciitis on physical examination.

Results: The study demonstrated that forefoot pronation on physical examination, high metatarsal pressure on the gait assessment, increasing time spent standing on hard surfaces, increased time spent walking, medium tenure at the plant, and an increased number of times getting in and out of the vehicle (for the truck/forklift drivers) increased the risk of presenting with plantar fasciitis. Rotation of shoes during the work week was found to reduce the risk of presenting with plantar fasciitis. Increased supervisor support showed a trend toward reducing the prevalence of plantar fasciitis.

Conclusions: Plantar fasciitis is relatively common in the manufacturing setting. These findings suggest several options for primary and secondary prevention strategies. Shoe rotation may be an effective strategy that may be used as either a primary or secondary strategy. The use of shoe orthoses with a medial longitudinal arch and metatarsal pad may be used as a preventive or treatment strategy. Work stations that decrease the percentage of time walking or standing on hard surfaces (eg, allowing workers to alternate between sitting and standing postures or providing cushioning mats for concrete surfaces) may lower the risk for plantar fasciitis.

PM R 2010;2:110-116

\section{INTRODUCTION}

Plantar fasciitis is a common cause of heel pain. It typically involves pain at the origin of the plantar fascia at the calcaneal insertion point as it is stretched during standing and walking activities. Plantar fasciitis can cause stabbing pain that is most intense with the first steps in the morning. With standing and walking activities, the pain related to plantar fasciitis normally decreases, but may return after long periods of standing or after getting up from a seated position.

Under normal circumstances, the plantar fascia acts like a biomechanical shock absorber, supporting the arch in the foot; but, if the tension on the plantar fascia exceeds the limits of the tissue, small tears can develop in the fascia. Repetitive tension and subsequent tearing can cause the fascia to become inflamed and painful. Plantar fasciitis is particularly common in runners, but is also noted among workers who stand for long periods [1].
R.A.W. PM\&R, University of Michigan, 2215 Fuller Rd. (117), Ann Arbor, Ml 48105. Address correspondence to: R.A.W.; e-mail: rawerner@med.umich.edu

Disclosure: 8B, grant support from the UAW/GM Health and Safety Board

N.G. PM\&R, University of Michigan, Ann Arbor, MI

Disclosure: 8A, grant support from the UAW/GM Health and Safety Board

A.H. PM\&R, University of Michigan, Ann Arbor, MI

Disclosure: 8A, grant support from the UAW/GM Health and Safety Board

N.W. Industrial Operations Engineering, University of Michigan, Ann Arbor, Ml Disclosure: 8B, grant support from the UAW/GM Health and Safety Board

W.M.K. Industrial Operations Engineering, University of Michigan, Ann Arbor, Ml Disclosure: 8A, grant support from the UAW/GM Health and Safety Board

Disclosure Key can be found on the Table of Contents and at www.pmrjournal.org

Submitted for publication September 18, 2009; accepted November 26. 
Risk factors for developing plantar fasciitis include older age (most common between the ages of 40 of 60), female gender, certain types of exercise that place high levels of stress on the heel and attached tissue (such as long-distance running, ballet dancing, and dance aerobics), faulty foot mechanics (flat feet, high arches, or an abnormal pattern of walking), obesity, occupations with prolonged standing, and improper shoes (eg, shoes that are thin-soled, loose, high heeled, or lacking arch support) [1-9].

There have been limited epidemiologic studies that focused on foot or ankle problems. Most of these studies had one or more methodological weaknesses [4]. Riddle et al (1) conducted a matched case-control study of plantar fasciitis and found significant associations between plantar fasciitis and body mass index (BMI), limited ankle dorsiflexion of the involved side, and spending the majority of the workday on the feet. The amount of time spent on feet was a dichotomous variable and was self-reported.

Ryan performed a cross-sectional study of musculoskeletal symptoms in supermarket workers $(n=513)$ [2]. The checkout department workers were mostly women and were observed to stand for long periods. They also had the highest prevalence of ankle/foot and lower limb complaints. Unfortunately, there was no physical examination of the foot to further assess the musculoskeletal problems.

deZwart et al $[5,6]$ evaluated the musculoskeletal complaints in the ankle and feet over 4 years in working men with respect to age and work demands. They found a significant prevalence increase of $2 \%$ of ankle and foot complaints in men who had jobs deemed to have heavy physical demands but only in the 40- to 49-year age strata. Dawson et al [8] conducted a population-based study of older (ages 50-70 years) women and found that those who reported foot pain had spent significantly more years doing work activities which required regular lifting. However, other work activities such as standing, lifting, walking, and kneeling were not found to be significant risk factors. Bergenudd et al [2] evaluated the feet in 574 men and women, age 55 years at the time of the physical examination, and did not find a difference in the prevalence of foot disorders based on the physical demands of the jobs. A low prevalence (less than 10\%) of ankle/foot musculoskeletal complaints was reported in some studies. Lemasters et al [9] inquired about musculoskeletal symptoms in the ankle and feet among carpenters, but they did not find a significant association.

Overall, the epidemiologic studies suggest that high BMI, prolonged standing, and high physical workload may be associated with ankle/foot musculoskeletal symptoms and plantar fasciitis. However, these studies did not adjust for floor surfaces and type of shoes, which have shown to affect fatigue and discomfort of the feet in laboratory studies $[4,10$ 12] Use of shock-absorbing "antifatigue" mats for many workers in assembly jobs have been instituted in many settings, but their effectiveness in reducing lower extremity musculoskeletal disorders, including plantar fasciitis, has not been well studied. The relative contributions of work-related risk factors (standing, walking on hard surfaces, carrying loads) versus personal risk factors (age, weight, BMI, foot biomechanics, type of shoe wear, and medical history) have not been well documented.

The goal of this study was to determine the relative contributions of work activity (time spent standing, walking, or sitting), floor surface characteristics, weight, BMI, age, foot biomechanics, and other demographic and medical history factors to the prevalence of plantar fasciitis in an automobile engine assembly plant.

\section{METHODS}

This was a cross-sectional study conducted at an automotive engine assembly plant in the northern United States. All salaried and hourly plant employees $(n=666)$, including those from assembly, machining, skilled trades, and administration were eligible to participate. Four hundred seven employees (61\%) were recruited into the study through use of fliers posted around the plant, a plant newsletter article, a 3-minute recruitment video that ran intermittently on the internal television system, and team meetings. Participation included completing a symptom questionnaire, undergoing a physical examination, and work exposure assessment based on a job analysis that focused on lower body posture. Data collection for the symptom questionnaire and physical examination portions of the study was performed during nonwork times, including breaks and before or after work hours. The job assessment was done during regular work hours through use of video taping of the job and pedometers. All subjects signed a consent form approved by the Institutional Review Board at the University of Michigan. Workers who agreed to participate in the study were given a shirt, and those who completed all aspects of the study received a $\$ 25$ participation incentive.

Participants completed a lower extremity symptom questionnaire that included a body diagram for pain, visual analog pain score, surgical history, and medications history. Demographics such as age, height, weight, and medical history for both generalized and lower extremity disorders were included in the questionnaire. Smoking history, exercise, other work activities, and fatigue levels were self-reported. Additionally, subjects completed a questionnaire focused on regional lower extremity pain that occurred within the last year and that lasted more than 1 week or occurred more than 3 times. They were asked to rate the regional pain using severity ratings of mild, moderate, and severe. The symptom questionnaire included a section of self-reported job satisfaction, supervisor support, and job insecurity as proposed by Karasek [13].

The subjects gave permission for their plant medical records to be reviewed for up to 2 years before the study. 
Treatment dates and medical diagnoses were abstracted from these medical records and compared with the questionnaire responses. Information from the medical records was used to determine prior history of lower extremity diagnoses and the relationship of these diagnoses to work or personal factors.

Subjects underwent a focused physical examination of the lower extremities to evaluate the hip, knee, and ankle joints as well as standing foot alignment, posture, and gait analysis. All physical examinations were performed by board-certified physicians in Physical Medicine and Rehabilitation or physical therapists. All examiners were trained to perform the evaluation in a standardized manner. Dynamic foot pressure recordings during gait were obtained on all subjects, with readings taken for 6 complete steps using the Tekscan Pressure Measurement System (Tekscan, Boston, MA). Pressure was recorded as pounds per square inch. The highest pressure recording at the heel, metatarsal heads, and great toe was used for analysis. The physical examination included observation for joint deformities and abnormalities such as rubor, increased temperature, skin changes, and varicose veins. Subjects who reported current localized symptoms in any lower extremity joint underwent additional tests including range of motion restrictions, joint laxity, and provocative diagnostic tests. Pes planus was defined as present if the inferior surface of the navicular bone was palpated and noted to be less than $2 \mathrm{~cm}$ above the floor while standing. Abnormal forefoot pronation was determined if the clinician saw excessive pronation during observed gait compared with standing.

In addition to the physical examination, a biomechanical evaluation of the shoe was also performed. The hardness of both the inside and outside of subjects' shoes was measured using a hand-held durometer (Durometer Model 409 Type C, Pacific Transducer Corp, Los Angeles, CA) with the highest of 3 measurements being recorded [14].

For the purpose of analysis, if a subject reported moderate or severe foot pain lasting more than 1 week or occurring at least 3 times in the past year and experienced tenderness to palpation at the insertion of the plantar fascia on the calcaneous, the subject was diagnosed with plantar fasciitis. "New" plantar fasciitis was defined as a worker with pain in the foot related to palpation of the plantar fascia at the calcaneous who did not report a history of plantar fasciitis in their medical history.

The evaluation of lower body posture/activity was performed on volunteers as they performed their regular jobs. All jobs were assessed and ergonomic exposures (ie, time spent in specific lower body postures) were calculated as percentages of a typical work day. The primary mode of exposure assessment was video analysis, with additional data collected via pedometer analysis, and interviews. In some instances, a time-weighting procedure was used to determine exposure to account for the job rotation system used by certain departments (eg, assembly-line jobs) at the study site. This procedure was used to determine the cumulative time spent during a typical work day in the following lower body postures: sitting, walking, standing, crouching, and carrying weighted objects. In addition the cumulative time spent working on the following surfaces was determined: concrete, asphalt, linoleum tile-on-concrete, antifatigue matting, and carpet. The details of the job assessment for assembly workers are described by Keyserling et al [15].

For workers who did not remain in a confined work area (ie, skilled trades, drivers, machinists, and administrative staff), pedometer recordings and end-of-day interviews were used exclusively to document lower body activities.

\section{Statistical Analysis}

A univariate analysis using $t$-tests (for continuous variables) or $\chi^{2}$ analysis (for categorical variables) was performed on subjects by job type (assembly, machining, driver, administrative, skilled trades) comparing them for baseline demographics, medical history, ergonomic exposures, psychosocial factors, discomfort ratings, and regional medical outcomes. Multivariate logistical regression was performed using plantar fasciitis, severe regional pain, and existing or new onset of joint dysfunction as the dependent variable to create a predictive model.

\section{RESULTS}

The subject data are presented in Table 1. A total of 407 workers completed the assessment. The average age of the cohort was 48.4 years \pm 10.3 (mean \pm standard deviation) with $73 \%$ having spent more than 20 years at the company. The cohort was $76 \%$ men with a mean BMI of $29.4 \pm 5.3$ (a person with a BMI higher than 30 is considered obese). Thirty-two subjects (8\%) met the case definition of plantar fasciitis. There were $23(6 \%)$ cases of new plantar fasciitis.

The logistic regression modeling of the prevalence of plantar fasciitis and new cases of plantar fasciitis are presented in Tables 2 and 3, respectively. The models are similar in the identification of risk factors. In Table 2, factors that increased the risk of presenting with plantar fasciitis included forefoot pronation on physical examination, high metatarsal pressure on the gait assessment, increasing time spent standing on hard surfaces, increased time spent walking, midrange tenure at the plant (4-7 years), and an increased number of times getting in and out of the vehicle (for the truck/ forklift drivers). Rotation of shoes during the work week was found to reduce the risk of presenting with plantar fasciitis. Increased supervisor support showed a trend toward reducing the prevalence of plantar fasciitis. Women were at higher risk $(\mathrm{OR}=3.4)$ of presenting with plantar fasciitis in the overall model, but not in the model of new cases.

Forefoot pronation was associated with a fourfold increase in the prevalence of plantar fasciitis, whereas high pressures at the metatarsal heads during gait increased the risk 2.7 
Table 1. Comparison of demographics, medical history, psychosocial variables, and work characteristics between workers with and without plantar fasciitis

\begin{tabular}{|c|c|c|c|}
\hline & $\begin{array}{c}\text { Subjects with } \\
\text { Plantar Fasciitis } \\
\qquad \mathbf{n}=\mathbf{3 2}\end{array}$ & $\begin{array}{c}\text { Subjects without } \\
\text { Plantar Fasciitis } \\
\qquad \mathbf{n}=\mathbf{3 7 5}\end{array}$ & $\boldsymbol{P}$ \\
\hline \multicolumn{4}{|l|}{ Demographics } \\
\hline$\%$ Workers with foot/ankle pain & $100 \%$ & $48 \%$ & .00 \\
\hline Age (years) & $48.6(9.5)$ & $48.4(10.3)$ & .91 \\
\hline Range & $28-62$ & $19-70$ & \\
\hline Body mass index & $31.2(6.9)$ & $29.2(5.2)$ & .04 \\
\hline Range & $23-52$ & $18-47$ & \\
\hline Gender (\% female) & $37 \%$ & $22 \%$ & .05 \\
\hline Weight (pounds) & $212.4(49.4)$ & $200.2(41.7)$ & .12 \\
\hline Range & $140-352$ & $115-350$ & \\
\hline \multirow[t]{3}{*}{ Tenure at job (at GM) } & $0-10$ years $19 \%$ & $0-10$ years $20 \%$ & .36 \\
\hline & $11-20$ years $19 \%$ & $11-20$ years $6 \%$ & \\
\hline & More than 20 years $63 \%$ & More than 20 years $74 \%$ & \\
\hline \multicolumn{4}{|l|}{ Medical history } \\
\hline Current smoker & $19 \%$ & $16 \%$ & .66 \\
\hline Exercise regularly & $59 \%$ & $67 \%$ & .37 \\
\hline Diabetes & $9 \%$ & $9 \%$ & .95 \\
\hline Rheumatoid arthritis & $9 \%$ & $5 \%$ & .30 \\
\hline Heel spur & $38 \%$ & $8 \%$ & .00 \\
\hline Prevalence of plantar fasciitis (prior diagnosis) & $28 \%$ & $7 \%$ & .00 \\
\hline \multicolumn{4}{|l|}{ Physical examination and shoe assessment } \\
\hline Peak metatarsal pressure (per square inch) & $70.3(14.6)$ & $63.7(13.9)$ & .01 \\
\hline Range & $42-99$ & 28-109 & \\
\hline Forefoot pronation (by observation) & $53 \%$ & $25 \%$ & .00 \\
\hline Pes plaus (by observation) & $31 \%$ & $25 \%$ & .40 \\
\hline Outer sole stiffness & $26.3(8.3)$ & $27.1(9.9)$ & .66 \\
\hline Range & $1-40$ & 4-92 & \\
\hline$\%$ Using shoe Rotation & $25 \%$ & $47 \%$ & .02 \\
\hline Any shoe insert (\%) & $56 \%$ & $32 \%$ & .005 \\
\hline Plant supplied shoe insert (\%) & $18 \%$ & $14 \%$ & .85 \\
\hline \multicolumn{4}{|l|}{ Psychosocial variables } \\
\hline Education level & & & .85 \\
\hline Did not complete high school & $0 \%$ & $2 \%$ & \\
\hline High school graduate & $39 \%$ & $39 \%$ & \\
\hline Some college & $39 \%$ & $35 \%$ & \\
\hline College grad or higher & $22 \%$ & $24 \%$ & \\
\hline Skill discretion (12-48) & $32.8(8.4)$ & $34.2(7.0)$ & .29 \\
\hline Range & $18-48$ & $12-48$ & \\
\hline Decision authority (12-48) & $32.8(8.2)$ & $33.2(8.1)$ & .75 \\
\hline Range & $20-48$ & $12-48$ & \\
\hline Supervisor support (4-16) & $10.9(2.8)$ & $11.9(2.2)$ & .03 \\
\hline Range & $4-16$ & $4-16$ & \\
\hline Job dissatisfaction & $0.27(0.21)$ & $0.22(0.21)$ & .18 \\
\hline \multicolumn{4}{|l|}{$0=$ more satisfied } \\
\hline \multicolumn{4}{|l|}{$1=$ less satisfied } \\
\hline Range & $0-0.67$ & $0-1$ & \\
\hline Coworker support (4-16) & $11.9(2.5)$ & $12.1(1.7)$ & .67 \\
\hline Range & $4-16$ & $4-16$ & \\
\hline Job insecurity (3-12) & $5.2(1.4)$ & $5.1(1.6)$ & .87 \\
\hline Range & $3-9$ & 3-12 & \\
\hline \multicolumn{4}{|l|}{ Job characteristics } \\
\hline \% Time walking or sidestepping & $28 \%(14.3)$ & $25 \%(12.4)$ & .18 \\
\hline Range & $6-64 \%$ & $6-64 \%$ & \\
\hline$\%$ Time on hard surfaces & $41.2 \%(23.5)$ & $34.2 \%(22.8)$ & .10 \\
\hline Range & $7-69 \%$ & $2-84 \%$ & \\
\hline $\begin{array}{l}\text { Number of times in/out vehicle } \\
\text { (drivers only } n=35 \text { ) }\end{array}$ & $116.0(76.6)$ & $80.7(45.1)$ & .15 \\
\hline Range & 39-195 & $33-195$ & \\
\hline
\end{tabular}


Table 2. Modeling the prevalence of plantar fasciitis, $n=372$

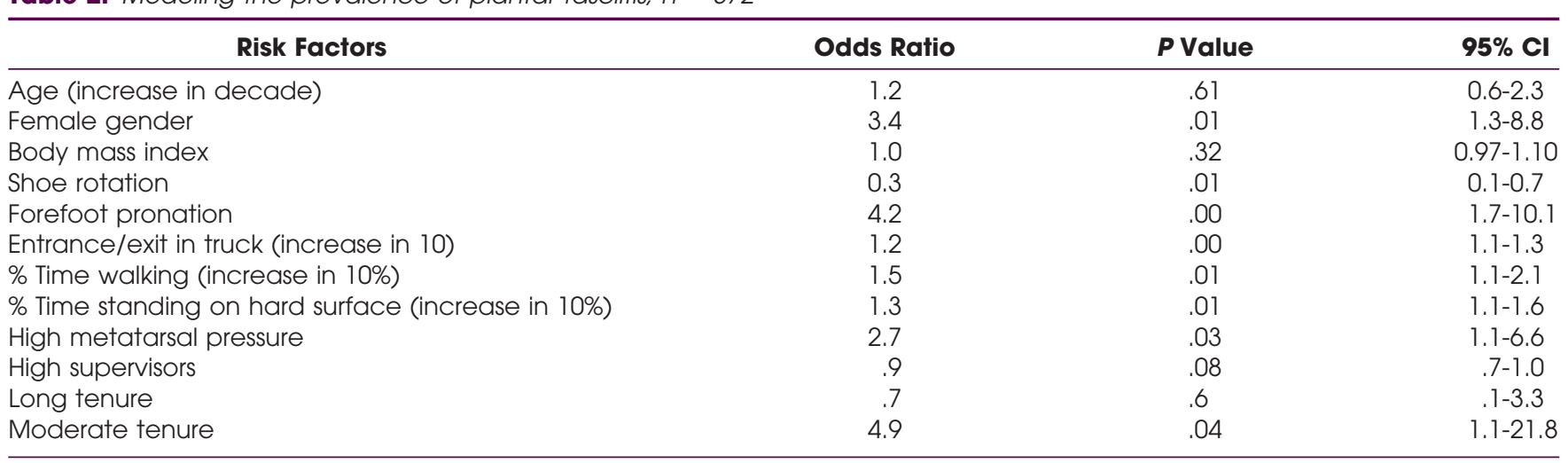

Pseudo $R^{2}=0.24$

times. A worker who spent 10\% more time at standing and walking activities had a $52 \%$ increased risk of presenting with plantar fasciitis. Workers who spent an additional 10\% of their time standing on hard surfaces (concrete, asphalt or linoleum tile on concrete) had a 30\% increase risk of plantar fasciitis. Rotation of shoes worn during the work week (wearing at least 2 different shoes) decreased the risk of presenting with plantar fasciitis by $72 \%$. Among truck and forklift drivers, for each additional 10 times they exited their vehicle, there was a $17 \%$ increased risk. Workers in the middle tertile of tenure at the plant had the highest risk of presenting with plantar fasciitis compared with the lower tertile of tenure. The highest tertile of tenure did not have an increased risk of plantar fasciitis.

The results were similar when analyzing new cases of plantar fasciitis. There was an increased risk associated with forefoot pronation, high metatarsal pressures, walking activities, standing on hard surfaces, and middle tertile of tenure that were of similar risk. In this model, job dissatisfaction was associated with a higher risk of presenting with plantar fasciitis. The composite score for job dissatisfaction has a range of 0 to 1 . For an increase of 0.1 on this scale, workers were $34 \%$ more likely to present with plantar fasciitis. For truckers, the number of times exiting their vehicle was still a significant risk factor. Rotation of shoes was still protective and workers who exercised regularly for longer periods were also found to be associated with a slightly lower risk.

\section{DISCUSSION}

The current study found that $8 \%$ of the cohort met the case definition of plantar fasciitis. Fifty-two percent had complaints of foot pain that lasted at least a week or recurred at least 3 times in the past 6 months and this is much higher than most other studies $[2,4]$. The study confirmed several of the known risk factors associated with the development of plantar fasciitis and was able to quantify the association based on quantitative job exposure of walking activities and standing on hard surfaces. The authors did not find a significant association between plantar fasciitis and older age or obesity. Gender was not a risk factor in the model of new cases of plantar fasciitis. The authors confirmed the association between plantar fasciitis and faulty foot mechanics (flat feet and forefoot pronation) and were able to establish an association between plantar fasciitis and high metatarsal pressures during gait. The authors did not find an association with the type of shoe worn, but did identify

Table 3. Modeling "new" plantar fasciitis, $n=346$

\begin{tabular}{|c|c|c|c|}
\hline Risk Factors & Odds Ratio & $P$ Value & $95 \% \mathrm{Cl}$ \\
\hline Age (increase in decade) & 1.6 & .27 & $0.7-3.4$ \\
\hline Female gender & 1.5 & .47 & $0.5-4.5$ \\
\hline Body mass index & 1.0 & .62 & $0.9-1.1$ \\
\hline Forefoot pronation & 5.4 & .00 & 1.9-15.7 \\
\hline Entrance/exit in truck (increase in 10) & 1.2 & .03 & $1.02-1.32$ \\
\hline$\%$ Time walking (increase in 10\%) & 1.5 & .01 & $1.1-1.2$ \\
\hline Job dissatisfaction (change of 0.1 on scale) & 1.3 & .02 & $1.05-1.70$ \\
\hline Frequent extended exercise & 0.9 & .02 & $0.93-0.99$ \\
\hline Long tenure & 1.0 & .98 & $0.1-8.0$ \\
\hline Moderate tenure & 8.3 & .05 & $1.05-65.5$ \\
\hline
\end{tabular}

Pseudo $\mathrm{R}^{2}=.29$. 
that rotation of shoes was protective against the development of plantar fasciitis.

Although several studies have documented the association between prolonged standing and walking activities to plantar fasciitis, the assessment of exposure has been very limited and typically was self-reported as a dichotomous variable [4]. The authors were able to accurately assess the percentage of time spent standing, walking, and sitting, as well as the type of surface these activities were performed on. This allowed the authors to determine that the risk of prolonged exposure had a linear relationship and predict the increased risk based upon the percentage of time spent walking in one job versus another job. In this cohort, an increase of $10 \%$ of the time spent walking translated into a $52 \%$ increased risk of plantar fasciitis. Similarly, the type of surface that a worker is standing on can impact the development of plantar fasciitis. An increase of $10 \%$ of their time on a hard surface is associated with 30\% increased risk.

Although increasing age, female gender, and obesity were associated with higher prevalence of plantar fasciitis in other studies, this was not consistently confirmed in this study $[1,5,6]$. The cohort the authors studied had a mean age of 48 and represents an older portion of the work force with three quarters of them having spent more than 20 years with this company. This may represent a "healthy worker effect" in which the sample the authors studied was successful workers who have not developed foot problems, whereas the workers who developed significant foot problems had left the work site. This is supported when the authors analyzed the association between plantar fasciitis and tenure at this company. The workers who were there the longest (the upper tertile) had no increase in the prevalence of plantar fasciitis compared with the newest hires, but the workers in the middle tertile of tenure were 5 times more likely to have the problem. The issue of gender may have been confounded by the distribution of gender in this cohort. Less than 25\% were women; this may have limited the authors' ability to identify a consistent association with gender. Obesity was not associated with a higher prevalence of plantar fasciitis but the cohort had a high mean BMI $\left(29.4 \mathrm{~kg} / \mathrm{m}^{2}\right)$. Having an older and heavier group of workers may have contributed to the high prevalence of symptomatic feet among this cohort compared with other studies in the literature [4].

Several authors have suggested that foot biomechanics may play a role in the development of plantar fasciitis, but none have been able to quantify the risk when controlling for work activity $[1,4]$. The authors demonstrate that observed and measured foot biomechanics are independent risk factors associated with the prevalence of plantar fasciitis. Workers with flat feet were not noted to be at a higher risk, but those workers who had forefoot pronation and high metatarsal pressure were associated with a higher prevalence of plantar fasciitis. Pronation increased the risk fourfold, whereas high metatarsal pressure increased the risk almost threefold. These are important risk factors because they are potentially responsive to preventive interventions. The use of shoe orthoses may be able to modify the risk by offloading the metatarsal heads through use of a metatarsal bar and appropriate support of the medial longitudinal arch to prevent pronation. The authors did assess whether workers had any orthotic devices, but this was not a significant predictor. It must be noted that the plant the authors studied did provide gel inserts for workers with foot complaints, but the insert did not have a significant medial longitudinal arch and did not have any metatarsal pads.

The finding of the protective association between shoe rotation and the prevalence of plantar fasciitis was somewhat of a surprise. The authors had hypothesized that the workers who chose to rotate their shoes would do so in response to experiencing foot pain and, as such, shoe rotation could have been a surrogate for foot pain and may have been identified as associated with a higher prevalence of plantar fasciitis. In fact, it was associated with a lower prevalence, which suggests that it is protective and, if used in response to experiencing foot pain, it is effective in reducing the foot problem. This could be a very effective means of prevention in the workplace.

Among the truckers and forklift drivers $(n=32)$, the authors found an increased prevalence of plantar fasciitis associated with higher number of times that the worker exited the vehicle. For every additional 10 times they exited the vehicle per shift, they were $18 \%$ more likely to present with plantar fasciitis. This is consistent with other studies that related higher prevalence with "high-impact" activities such as ballet dancing, dance aerobics, and running, in which there is repetitive loading of the plantar fascia [4]. A potential intervention would be encouraging these drivers to use shoes or inserts that provide effective cushioning and shock absorption.

The strength of this study is the detailed assessment of relevant job exposures across an entire assembly plant, including assembly line workers, skilled trade workers, and office workers. Although not every individual was assessed, every job category was assessed to provide each worker with an individualized ergonomic assessment of their exposure to standing, walking, the type of surface they worked on, as well as any loading activities. The diagnosis was based on a physical examination in combination with a symptom questionnaire. The authors had a high rate of participation for the entire plant, $72 \%$ for assembly line workers and skilled trades, and more than $50 \%$ for the managerial workers. The exposures were quite varied with some workers having primarily sedentary jobs, whereas many had jobs requiring prolonged standing.

The limitations of the study include a modest sample size. The cohort was older, heavier, and predominantly male when compared with many of the studies reported in the literature so may not be generalized to all workers. 
Assessment of the skilled trade workers was more challenging because of their movement throughout the plant and variation in the nature and duration of lower body exposures from day to day. As a cross-sectional study, the authors can only ascertain associations and may not prove causality. A longitudinal study would be necessary to demonstrate causation.

\section{CONCLUSIONS}

Plantar fasciitis is relatively common in the manufacturing setting. An increased risk is associated with forefoot pronation, high metatarsal pressure, increased time spent standing on hard surfaces, increased time spent walking and an increased number of times getting in and out of the vehicle (for the truck/forklift drivers). Rotation of shoes was found to reduce the risk of presenting with plantar fasciitis. These findings suggest several options for primary and secondary prevention strategies. Shoe rotation seems to be an effective strategy that may be used as either a primary or secondary strategy. The use of shoe orthoses with a medial longitudinal arch and metatarsal pad may be used in the same way. Work stations that decrease the percentage of time walking or standing on hard surfaces (eg, allowing workers to alternate between sitting and standing postures, or providing cushioning mats for concrete surfaces) may lower the risk for plantar fasciitis.

\section{REFERENCES}

1. Riddle DL, Pulisic M, Pidcoe P, Johnson RE. Risk factors for plantarfasciitis: A matched case-control study. J Bone Joint Surg 2003;85a: 872-877.

2. Bergenudd H, Lindgarde F, Nilsson B. Prevalence and coincidence of degenerative changes of the hands and feet in middle age and their relationship to occupational work load, intelligence, and social background. Clin Orthop 1989;239:306-310.

3. Ryan GA. The prevalence of musculo-skeletal symptoms in supermarket workers. Ergonomics 1989;32:359-371.
4. d'Souza J, Franzblau A, Werner RA. Review of epidemiological studies on occupational factors and lower extremity musculoskeletal and vascular disorders and symptoms. J Occup Rehabil 2005;15: 129-165.

5. deZwart BCH, Broersen JPJ, Frings-Dresen MHW, van Dijk FJH. Repeated survey on changes in musculoskeletal complaints relative to age and work demands. Occup Environ Med 1997;54:793799.

6. de Zwart BC, Broersen JP, van der Beek AJ, Frings-Dresen MH. Occupational classification according to work demands: An evaluation study. Int J Occup Med Environ 1997;10:283-295.

7. Merlino LA, Rosecrance JC, Anton D, Cook TM. Symptoms of musculoskeletal disorders among apprentice construction workers. Appl Occup Environ Hyg 2003; 18:57-64.

8. Dawson J, Thorogood M, Marks S, et al. The prevalence of foot problems in older women: A cause for concern. J Public Health Med 2002;24:77-84.

9. Lemasters GK, Atterbury MR, Booth-Jones AD, et al. Prevalence of work related musculoskeletal disorders in active union carpenters. Occup Environ Med 1998;55:421-427.

10. Cham R, Redfern MS. Effect of flooring on standing comfort and fatigue. Hum Factors 2001;43:381-391.

11. Redfern MS. Influence of flooring on standing fatigue. Hum Factors 1995;37:570-581

12. Redfern MA, Cham $R$. The influence of flooring on standing comfort and fatigue. AIHAJ 2000;61:700-708

13. Karasek R, Brisson C, Kawakami N, Houtman I, Bongers P, Amick B. The job content questionnaire (JCQ): an instrument for internationally comparative assessments of psychosocial job characteristics. J Occup Health Psych 1998;3:322-355.

14. Chiou SY, Bhattacharya A, Succop PA. Effect of workers' shoe wear on objective and subjective assessment of slipperiness. Am Ind Hyg Assoc J 1996;57:825-831.

15. Keyserling WM, Wiggermann N, Werner RA, Gell N. Inter-worker variability in lower body postures during assembly line work: implications for exposure assessment. Journal of Occupational and Environmental Hygiene. In press.

This CME activity is designated for 1.0 AMA PRA Category 1 Credit ${ }^{\mathrm{TM}}$ and can be completed online at me.aapmr.org. Log on to www.me.aapmr.org, go to Lifelong Learning (CME) and select Journal-based CME from the drop down menu. This activity is FREE to AAPMER members and $\$ 25$ for non-members. 